\title{
EMPRENDEDORISMO CON JOVENES PRIVADOS DE LIBERTAD
}

Marcos Lavandera $(*)$

\section{RESUMEN}

Este artículo pretende socializar una experiencia rica, intensa y práctica, de estudiantes y docentes de una facultad de ingeniería trabajando competencias emprendedoras en contexto de encierro. Esta iniciativa se enmarca en el Proyecto de Extensión "Para Emprendedores" de la Facultad de Ingeniería de Olavarría de la Universidad Nacional del Centro de la Provincia de Buenos Aires, Argentina.

Uno de los objetivos de este abordaje es brindar una formación de calidad, ampliar y modernizar la oferta de Formación Profesional, priorizando la línea de Educación para el trabajo a través del trabajo cooperativo y el emprendedorismo en el marco de la economía social en jóvenes privados de libertad. La experiencia se desarrolla en el Centro Cerrado Leopoldo Lugones de Azul, con jóvenes menores de edad que cumplen procesos judiciales o condenas. Se brindó capacitación en Emprendedorismo con estudiantes de ingeniería.

Palabras clave: Competencias emprendedoras - Emprendedorismo - Contexto de Encierro - Jóvenes -

\section{INTRODUCCIÓN}

Este artículo pretende socializar una experiencia rica, intensa y práctica, de estudiantes y docentes de una facultad de ingeniería trabajando competencias emprendedoras en contexto de encierro. Esta iniciativa se enmarca en el Proyecto de Extensión "Para Emprendedores" de la Facultad de Ingeniería de Olavarría de la Universidad Nacional del Centro de la Provincia de Buenos Aires, Argentina.

Uno de los objetivos de este abordaje es brindar una formación de calidad, ampliar y modernizar la oferta de Formación Profesional, priorizando la línea de Educación para el trabajo a través del trabajo cooperativo y el emprendedorismo en el marco de la economía social en jóvenes privados de libertad. La experiencia se desarrolla en el Centro Cerrado Leopoldo Lugones de Azul, con jóvenes menores de edad que cumplen procesos judiciales o condenas. Se brindó capacitación en Emprendedorismo con estudiantes de ingeniería, logrando en ellos resultados concretos que alcanzan también a las instituciones participantes.

Esta iniciativa se realiza como una de

(*) Ingeniero Químico y Magister en Administración de Negocios. Secretario de Extensión, Vinculación y Transferencia de la Facultad de Ingeniería de Olavarría. Jefe de Trabajos Prácticos Ordinario, Dpto. de Ing. Industrial. Integrante del Consejo Directivo de la Cátedra Abierta Latinoamericana de Innovación y Emprendedorismo (CALIYE). Director del Proyecto de Extensión "Para Emprendedores" y Director del Centro de Desarrollo Emprendedor e Innovación (CDEI) de la Facultad de Ingeniería de Olavarría. Universidad Nacional del Centro de la Provincia de Buenos Aires (UNICEN). Argentina.E-mail: marcos@fio.unicen.edu.ar 
las actividades del Proyecto de Extensión "Para Emprendedores" [1] y por solicitud del Programa para el Abordaje de Problemáticas Sociales y Relaciones con la Comunidad de la Defensa Pública del Departamento Judicial Azul.

El Proyecto de Extensión "Para Emprendedores" se alinea con una fuerte definición política institucional de la Facultad de Ingeniería sobre la extensión universitaria $^{[2]}$ y la formación humanística ${ }^{[3]}$ de los ingenieros y tiene como Objetivo General "Fomentar la cultura emprendedora facilitando la generación de nuevos emprendimientos $y$ fortaleciendo los ya existentes, a través de un espacio inclusivo, colaborativo, interdisciplinario e interinstitucional, dentro del ámbito universitario, para el apoyo a emprendedores que contribuyan al desarrollo económico regional con igualdad de oportunidades y equidad social". Algunos de sus objetivos específicos son: Fortalecer la red interinstitucional en relación a la temática emprendedora; Acercar a los estudiantes a las diferentes realidades socioeconómicas; Favorecer a la inclusión social fomentando el espíritu emprendedor en sectores vulnerables; Contribuir a la formación de una masa crítica de actores universitarios y no universitarios, de manera coordinada y metodológica, para el apoyo a emprendedores. En línea con lo establecido por el Consejo Federal de Decanos de Ingeniería (CONFEDI) en el "3er. Taller s/ Desarrollo de Competencias en la Enseñanza de la Ingeniería Argentina” año $2006^{[4]}$ y adoptado por la Asociación Iberoamericana de Entidades de Educación en Ingeniería ASIBEI, que indica que los ingenieros deben tener la competencia para "actuar con espíritu emprendedor", conocida como la "competencia 10".

La ejecución de esta iniciativa fue acompañada económicamente por las Universidad Nacional del Centre de la Provincia de Buenos Aires, a través de la Convocatoria a Proyectos de Extensión ${ }^{[5]}$ y el Programa Nacional de Voluntariado Universitario de la Secretaría de Políticas Universitarias del Ministerio de Educación de la Nación ${ }^{[6]}$.

Se podrían introducir los aspectos socioeconómicos, políticos e institucionales que llevan a los jóvenes a este tipo de Centros, pero no es motivo de este artículo profundizar sobre esto. Si es de interés, en cambio, rescatar que existe un sistema muy complejo de desigualdades, discriminación, estereotipos, falta de oportunidades, estrategias ineficientes, escasez de recursos y de idoneidad, que no contribuyen demasiado a impedir que algunos jóvenes lleguen a estos lugares, y luego en ellos tampoco encuentren herramientas de reinserción social. Los jóvenes universitarios, muchos de ellos provenientes de hogares con necesidades básicas insatisfechas, pero con otras herramientas culturales o sociales, pueden hacer una muy importante contribución al proyecto de vida de sus pares "encerrados". En ese sentido se perfila esta propuesta.

\footnotetext{
${ }^{[1]}$ http://pepe.fio.unicen.edu.ar/

${ }^{[2]}$ Res. C. A. Fac. Ing. No 116/11. Convocatorias a Proyectos de Extensión.

${ }^{[3]}$ Res. C. A. Fac. Ing. No 101/04. Actividades de Formación Social y Humanística

${ }^{[4]}$ Primer Ačuerdo sobre Competencias Genéricas "3er. TALLER s/ DESARROLLO DE COMPETENCIAS EN LA ENSENAANZA DE LA INGENIERÍA ARGENTINA”- Experiencia Piloto en las terminales de Ing. Civil, Electrónica, Industrial, Mecánica y Química. Villa Carlos Paz, 14 y 15 de agosto 2006 3er. INFORME. AGOSTO 2006.

${ }^{[5]} \mathrm{http}: / /$ extension.unicen.edu.ar/blog/?cat=427

${ }^{[6]}$ http://portales.educacion.gov.ar/spu/noticias/comienza-la-inscripcion-a-la-10-convocatoria-anual-del-programa-voluntariado-universitario/
} 


\section{DESCRIPCIÓN Y TRATAMIEN- TO DEL CASO}

Como toda acción emprendedora institucional, es importante establecer redes que puedan darle sustento y permanencia al proyecto. Así, se trabajó en conjunto con el Proyecto Jóvenes Emprendedores $\mathrm{Ru}-$ rales del Ministerio de Agricultura, Ganadería y Pesca de la Nación - PROSAP - UCAR, el Programa para el Abordaje de Problemáticas Sociales y Relaciones con la Comunidad, Defensa Pública Departamento Judicial Azul, el Centro de Referencia de Azul de la Secretaría de Niñez y Adolescencia de la Provincia de Bs. As. y la Defensoría $\mathrm{N}^{\circ} 1$ Fuero de Responsabilidad Penal Juvenil de Azul.

Asíse pudo llevar adelante un ciclo de encuentros que permitió cumplir con los objetivos en un mutuo aprendizaje con alto contenido solidario y de compromiso social.

Se trabajó con 17 jóvenes en 11 visitas sistematizadas en un día y hora determinados en la semana, logrando instalar el "día del Emprendedorismo" (así lo llamaron). Se generó un espacio en el que 6 estudiantes de ingeniería, durante 2 horas, compartían los saberes en un claro "modelo de rol" positivo.

Como ingenieros, los integrantes del equipo de la facultad propusieron una planificación de todos los encuentros, sus contenidos, metodologías y recursos necesarios. La realidad los llevó exactamente por otros caminos, la improvisación coherente, la búsqueda de otros recursos, otras técnicas, otra forma de comunicarnos y muy importante, situarnos en otro lugar "del poder", ya no desde el brindar conocimiento sino desde el "compartir conocimientos". Se pretendió iniciar el trabajo en la metodología taller

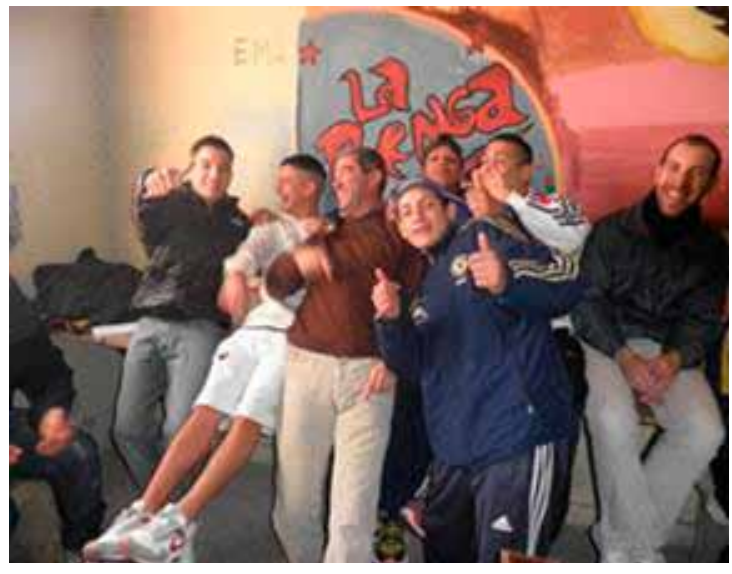

Foto 1: Acuerdo para trabajar Emprendedorismo.

incluyendo exposiciones con soporte power point, duró un solo encuentro, muchos de los jóvenes no saben leer bien de corrido y no podían seguir la temática. Hubo que cambiar rápidamente y trabajar conceptos y contenidos desde el "hacer", con alta participación de los internos y el equipo de la facultad acompañando el proceso.

Muchas dificultades se presentaron, una de las más importantes fue la alta rotación de jóvenes. No se pudo conformar un grupo permanente y esto quitó eficacia al proyecto; no obstante se pudo abordar temas como: autoconocimiento, competencias emprendedoras, comunicación, trabajo en equipo, ideas-oportunidades-proyectos, acceso a los recursos, tipo de emprendedores, proceso emprendedor y redes de contactos, conceptos trabajados desde la ética y buen uso de estas herramientas. También, emprendedores de la comunidad contaron sus experiencias como estrategia para reforzar los conceptos y desde ahí se trataron de concretar emprendimientos a través de los talleres de oficios que dispone la institución. Primero se realizó un taller en el que se produjeron los cuadernillos con los que iban a trabajar, a través de una "línea de producción" en la que se pudieron mostrar todas las partes integrantes del proce- 
so. Luego, en el $9^{\circ}$ encuentro, se empezaron a estudiar los posibles proyectos que los jóvenes podían llevar a cabo dentro del Centro, a través de la técnica de Brainstorming se hizo una preselección de acti- vidades emprendedoras y se eligieron tres que eran de interés común: elaboración de pastas caseras, huerta, manualidades - artesanías. Finalizada la selección se designó un responsable para cada una de ellas.

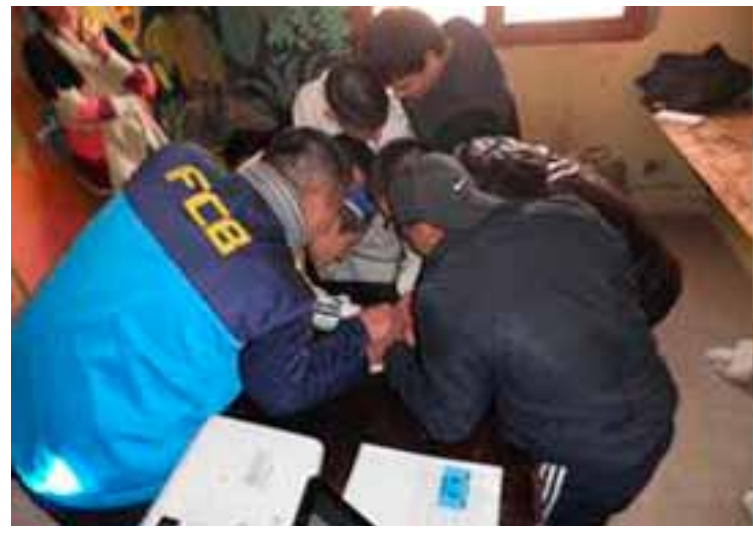

Foto 2: Trabajo en Equipo.

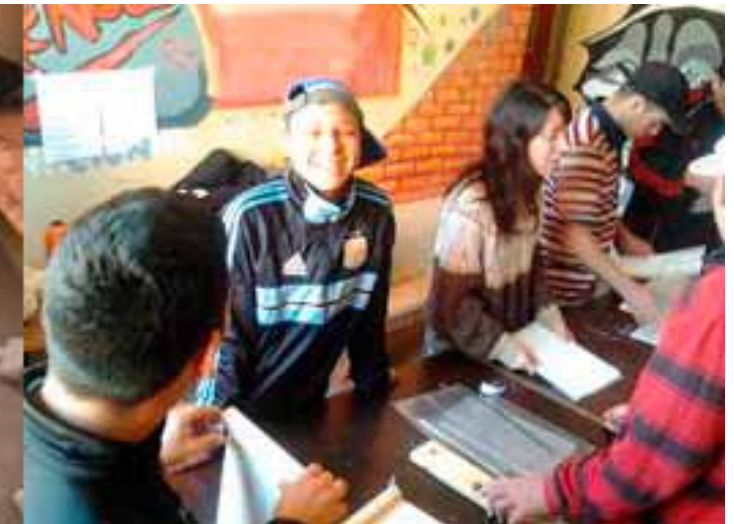

Foto 3: Producción de Cuadernillos.
Recién en el $10^{\circ}$ Encuentro, con los proyectos definidos, se terminaron de identificar los recursos necesarios para la elaboración de los productos. Seguido de esto, se recorrieron las instalaciones junto a integrantes del PEPE, un joven del establecimiento y el director del instituto. Se visitaron los posibles lugares donde se podrían desarrollar los emprendimientos, los equipos/herramientas con los que contaban y las refacciones que necesitarían para que sean aptos para la elaboración de los productos definidos en las actividades. En el taller de carpintería arreglaron mobiliario escolar y retomaron los talleres de panadería, pastas y huerta. La institución quedó comprometida a darle continuidad a estos proyectos y sobre fin de año se realizó la reunión final con entrega de diplomas, un hecho de altísimo valor emotivo y de mucho significado para los jóvenes que recibían por primera vez un certificado, y entregado por una universidad.

\section{RESULTADOS y DISCUSIÓN}

Resulta imprescindible destacar y valo- rar que 5 de estos 11 encuentros fueron desarrollados íntegramente por los estudiantes de la facultad, sin presencia de docentes, poniendo en práctica y evidenciando sus propias competencias emprendedoras.

Se alcanzaron los objetivos específicos del proyecto a través de estos 11 encuentros con 6 estudiantes de ingeniería y 17 jóvenes privados de libertad.

Si bien en ingeniería "gustan" los resultados cuantitativos, este es un caso concreto de muchos resultados cualitativos. Para mostrarlo, valen las palabras del Defensor General del Departamento de Azul en nota enviada al Sr. Subsecretario de Niñez y Adolescencia de la Prov. De Buenos Aires, "se ha incentivado el ambiente emprendedor en jóvenes en conflicto con la ley penal, a través de la intervención de este proyecto conjunto donde los jóvenes puedan adquirir conocimientos y herramientas para llevar a cabo emprendimientos tanto dentro como fuera del contexto de encierro. La capacitación en Emprendedorismo cabe como una 
dimensión del proyecto de vida que debemos promover en jóvenes que carecen del mismo (en medio de las carencias afectivas y socioeconómicas que caracterizan su vida cotidiana, el futuro no puede pensarse más allá de la inmediatez) o que está asociado a formas y contenidos que la sociedad considera y legisla como inadecuados para una convivencia social. Esta capacitación introduce y complementa la tarea de otras instituciones de niñez y adolescencia involucradas en los procesos penales de estos jóvenes, en la posibilidad de pensar y diseñar este proyecto de vida. Los estudiantes que participaron directamente del proyecto reconocen la incorporación de competencias que la academia no les proporciona desde lo curricular y entienden la extensión universitaria como herramienta para su adquisición. Al respecto, vale compartir algunos testimonios de los estudiantes.

-Sebastián: "Siento que fue una experiencia muy linda y muy rica en todo sentido. El hecho de estar trabajando con chicos cuya realidad es completamente distinta a la nuestra te hace ver cosas que en el día a día, no ves, o no prestas atención. Estos chicos emprenden como cualquier persona, pero su entorno los limita, viven de cerca la droga, los delitos, los maltratos familiares, la muerte. Sin embargo ellos estaban abi con nosotros, queriendo aprender a emprender, con otros fines y necesidades que quizá nosotros no perseguimos. Ellos ven en el emprendedorismo una reinserción en la sociedad y eso creo que es lo bueno. En conclusión, siento que esta experiencia nos sirvió a todos, a nosotros, "los de afuera", para ver otras realidades, otras necesidades. $Y$ a ellos mucho más aún, para conocer y aprender otro estilo de vida, que es emprender".

-Renzo: “Lugones" fue una experiencia emprendedora distinta a cualquiera que baya- mos hecho antes. Una realidad dura con historias de vida difíciles que se comparten y en la cual uno trata de obtener lo mejor de eso y volverlo a favor de los pibes. Son emprendedores de una economía distinta donde la principal traba no es el dinero ni los recursos, sino el encierro y las limitaciones del lugar. Pero de todo eso con los compañeros del PEPE tratamos siempre de buscarle el lado positivo, y anunciarles las posibilidades que tienen por estar en donde están y recordarles que su recurso más valioso es el "tiempo". Son jóvenes y si bien se equivocaron en las decisiones que han tomado es importante que todos les demos una nueva oportunidad y, es nuestra responsabilidad como ciudadanos ser parte de ese cambio y no liberarlos a la calle como cuando entraron, sino que este lugar se convierta en un aprendizaje de valores y herramientas para que el día de mañana estos pibes tengan la oportunidad que la sociedad no les dio. En lo personal estoy agradecido de haber participado de estos encuentros y tener la posibilidad de poder compartir conocimientos que me fueron brindados en la carrera con jóvenes de una realidad distinta. Estas experiencias nos ayudan a recordar lo afortunados que somos, por tener la familia que tenemos, por las posibilidad que nos dieron, por la educación que se nos brinda y por vivir la realidad que nos toca. Por esto, en mi opinión, debería incentivarse a la participación de más jóvenes en estos proyectos y que puedan compartir con otros su experiencia y contagiar, para que los conocimientos que adquirieron en la universidad sean re transmitidos a quien les dio la posibilidad de adquirirlos, la sociedad."

Los jóvenes internos, a su vez, reconocieron en este proyecto una forma distinta de aprender y la posibilidad de identificar otras formas de vida. Encontraron en los estudiantes personas con quien "ranchear" con otros temas y experiencias. 
La responsable del Programa para el Abordaje de Problemáticas Sociales y Relaciones con la Comunidad de la Defensa Pública del Departamento Judicial Azul, concluye, "En principio, el derecho a la educación y al trabajo son derechos humanos inalienables que el Estado debe garantizar a todos los seres humanos. La restricción del derecho a la libertad ambulatoria por condena judicial no solo no cercena estos derechos sino que obliga al Estado a hacer efectivos los medios para asegurar su acceso a quienes han sido condenados o se encuentren en proceso judicial por la comisión de delitos. $\mathrm{O}$ sea, en principio, es una garantía a la que la Universidad y la Defensa Pública, en tanto instituciones estatales, estamos obligados.”

\section{CONCLUSIONES}

A través de este proyecto la Facultad de Ingeniería de la UNICEN ha comenzado a transitar un camino interesante, de alto compromiso social y con múltiples facetas que permiten incorporar las más diversas Competencias Emprendedoras.

Con estas iniciativas se "rompe" con la lógica académica tradicional, el proceso de enseñanza - aprendizaje se vuelve vivencial y más dirigido al aprendizaje. Las reflexiones sobre el contexto socioeconómico y el ámbito de desarrollo profesional, así lo reflejaban, los estudiantes de ingeniería tomaron contacto directo con realidades desconocidas, hasta ese momento ajenas, ahora esa mirada está en debate e interpela el rol social de la universidad en general y de la ingeniería en particular. La Innovación Social y la Innovación Pública empiezan a visualizarse como herramientas de transformación y la ingeniería como protagonista cultural y tecnológica.

Debemos reforzar la valoración de la extensión universitaria como un proceso muy potente, ideológica y acadé-

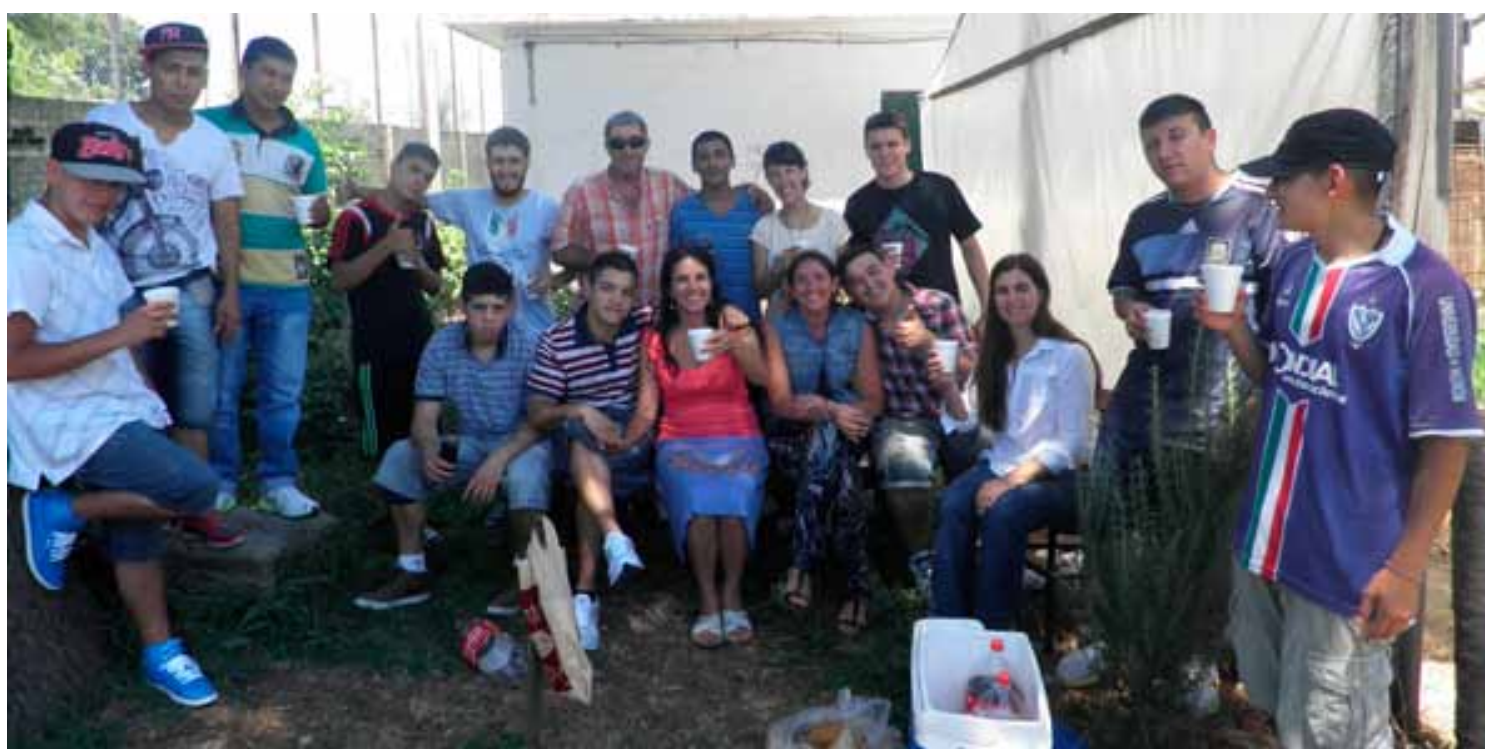

Foto 4: Entrega de Certificados.

micamente, que como estrategia institucional, contribuye a la formación por competencias, a la vez que se cumple con el rol social de la universidad más allá de la estricta práctica docente en el aula. 


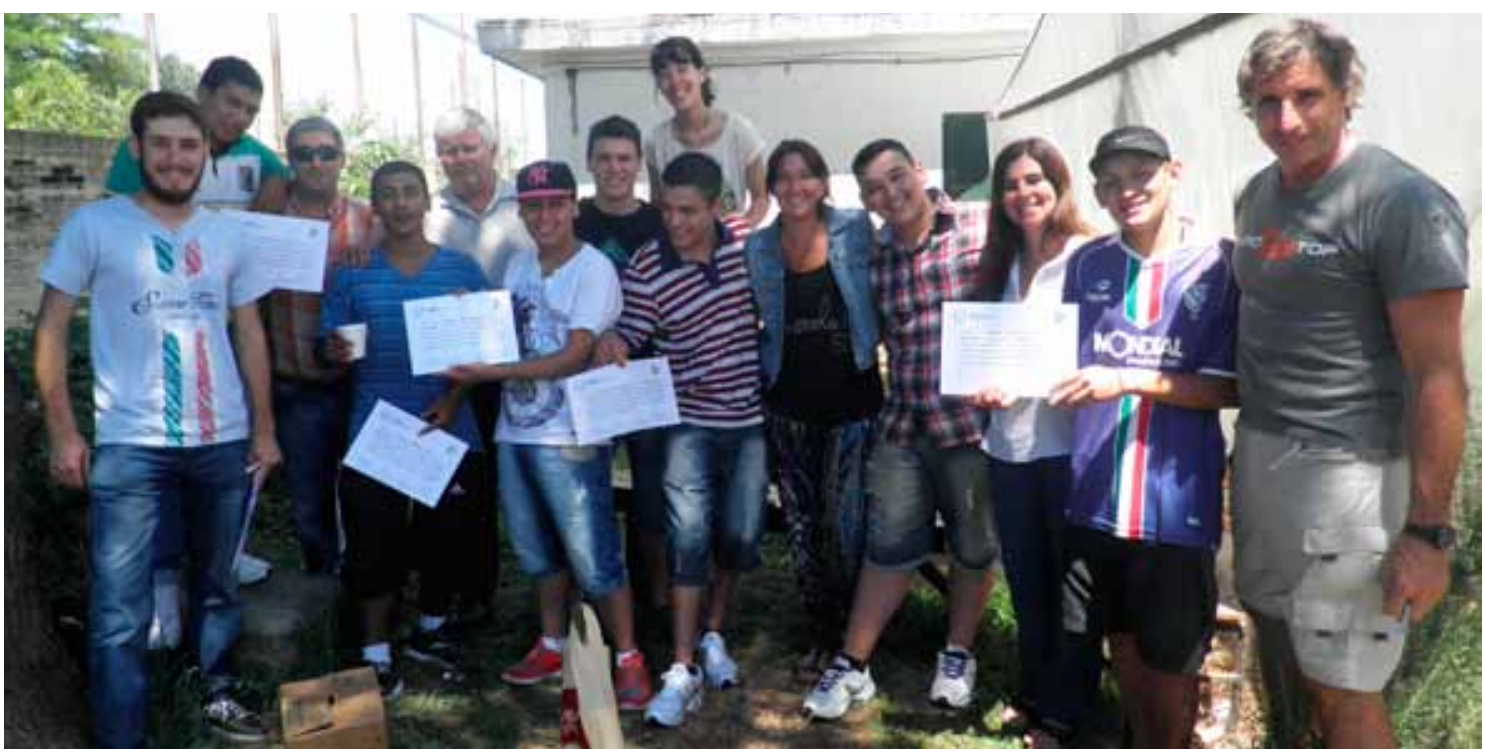

Foto 5: Entrega de Certificados.

En este sentido, se debe continuar trabajando en la formación social de los profesionales, más allá de su ámbito de actuación, para poder intervenir en las causas que hacen al mejoramiento de la calidad de vida de los ciudadanos y así disminuir las consecuencias de la vida como las que el equipo de estudiantes y docentes de la Facultad de Ingeniería pudo conocer. La Ingeniería tiene mucho para aportar en este sentido, resolver cuestiones de la vida cotidiana que impactan directamente en la construcción se sociedades más iguales.

Seguramente esta experiencia abre deba- tes, genera ideas y contradicciones. Si es así, se ha cumplido otro objetivo, implícito, del autor.

\section{AGRADECIMIENTOS}

Este trabajo no se hubiese podido realizar sin la participación activa, comprometida y solidaria de los estudiantes, Paula Bertolli, Luciana Mentasti, Mariana Leal, Emilia Vales, Renzo Barrionuevo, Sebastián Coria y Pablo Iarussi; y las funcionarias, Lic. Pilar Gonzalez, Dra. Laura Serradel y Sra. Gabriela Mattina 